\title{
Immunohistochemical detection of lymph node microinvolvement in node-negative gastric cancer
}

\author{
Youichi Kikuchi, Atsuo Tsuchiya, Yosiro Ando, Tsuneyuki Yoshida, and Seitchi Takenosita \\ Second Department of Surgery, Fukushima Medical University, Hikarigaoka 1, Fukushima 960-1247, Japan
}

\begin{abstract}
:
Background. Despite curative resection of the primary tumor with extensive dissection of lymph nodes, some patients with node-negative gastric cancer die of local relapse or metastatic disease. Microinvolvement of regional lymph nodes may play an important role in the prognosis.

Methods. To evaluate the incidence and prognostic implications of regional lymph node microinvolvement in nodenegative gastric cancer, we retrospectively analyzed tissue samples from 51 patients operated on for primary gastric cancer. They had tumors that invaded beyond the muscularis propria, but without metastasis to the lymph nodes, shown by the conventional H\&E staining method. The regional lymph nodes were examined immunohistochemically, using monoclonal antibodies against cytokeratin.

Results. Microinvolvement was found in $4.8 \%$ of lymph nodes (67/1390) and in $43.2 \%$ of the patients $(22 / 51)$. The clinical outcome of the patients with microinvolvement was not significantly different from those without it. However, no patient died in the no-microinvolvement group, while three patients in the microinvolvement group died of recurrence.

Conclusion. The incidence of microinvolvement in conventionally negative lymph nodes cannot be ignored, and detecting microinvolvement may be important for predicting recurrence of gastric cancer.
\end{abstract}

Key words: gastric cancer, regional lymph node, microinvolvement, cytokeratin, prognosis

\section{Introduction}

Gastric cancer is one of the leading causes of cancer death in Japan. Despite curative resection of the primary tumor with extensive dissection of lymph nodes, over half of all patients with advanced gastric cancer die

Offprint requests to: Y. Kikuchi

Received for publication on April 26, 1999; accepted on Sept. 14, 1999 of local relapse or metastatic disease. Lymph node status, and depth of invasion in the gastric wall, are the most valuable prognostic factors in gastric cancer. A multivariate analysis has suggested that lymph node status in gastric cancer is the most independent prognostic factor in terms of recurrence and survival [1]. Recurrence was frequent in node-positive gastric cancer patients. Adachi et al. [2] reported that the 10-year survival rate of patients with node-negative tumors was $93.4 \%$, but some node-negative gastric cancer patients died of recurrence. Currently, the method used to detect micrometastasis is not adequate. Sometimes it is not possible to identify any malignant cells by hematoxylin and eosin (H\&E) staining.

Intracytoplasmic filaments, composed of cytokeratin proteins that constitute part of the cytoskeleton, are exclusively present in cells that display epithelial differentiation. They serve as markers for epithelial cells [3-5]. Since cytokeratin-positive cells are normally not present in the lymph node, such cells in regional lymph nodes are considered to be foreign cells, suggesting metastasis. Immunohistochemical localization with cytokeratin in the lymph node was shown to identify micrometastases that had been missed on routine tissue staining in patients with colorectal cancer [6,7], breast cancer [8], and gastric cancer [9-11].

This study was performed to determine the usefulness of immunohistochemical staining for the detection of occult metastases in regional lymph nodes, and to assess the clinical implications in regard to survival in patients with gastric cancer.

\section{Patients and methods}

This retrospective study included 51 Japanese patients with primary gastric cancer, all of whom had undergone gastric resection with lymph node dissection at the Second Department of Surgery, Fukushima Medical 
Table 1. Relationship between microinvolvement and clinicopathological factors in patients with gastric cancer

\begin{tabular}{|c|c|c|c|c|}
\hline \multirow[b]{2}{*}{ Characteristics } & \multirow[b]{2}{*}{ No. of patients } & \multicolumn{2}{|c|}{ Microinvolvement } & \multirow[b]{2}{*}{$P$ value } \\
\hline & & $(-) n=29$ & $(+) n=22$ & \\
\hline \multicolumn{5}{|l|}{ Age (years) } \\
\hline$<50$ & $n=7$ & 3 & 4 & NS \\
\hline $50-70$ & $n=31$ & 20 & 11 & \\
\hline$>70$ & $n=13$ & 6 & 7 & \\
\hline \multicolumn{5}{|l|}{ Gender } \\
\hline Male & $n=36$ & 22 & 14 & NS \\
\hline Female & $n=15$ & 7 & 8 & \\
\hline \multicolumn{5}{|l|}{ Location } \\
\hline Upper third & $n=12$ & 5 & 7 & NS \\
\hline Middle third & $n=18$ & 12 & 6 & \\
\hline Lower third & $n=21$ & 12 & 9 & \\
\hline \multicolumn{5}{|c|}{ Maximum tumor size (mm) } \\
\hline$<40$ & $n=28$ & 19 & 9 & $P<0.05$ \\
\hline $40-80$ & $n=19$ & 10 & 9 & \\
\hline$>80$ & $n=4$ & 0 & 4 & \\
\hline \multicolumn{5}{|l|}{ Macroscopic type } \\
\hline Type 0 & $n=8$ & 7 & 1 & NS \\
\hline Type 1 & $n=6$ & 2 & 4 & \\
\hline Type 2 & $n=19$ & 12 & 7 & \\
\hline Type 3 & $n=11$ & 6 & 5 & \\
\hline Type 4 & $n=3$ & 1 & 2 & \\
\hline Type 5 & $n=4$ & 1 & 3 & \\
\hline \multicolumn{5}{|c|}{ Depth of tumor invasion } \\
\hline $\mathrm{t} 2$ & $n=39$ & 26 & 13 & $P<0.05$ \\
\hline $\mathrm{t} 3, \mathrm{t} 4$ & $n=12$ & 3 & 9 & \\
\hline \multicolumn{5}{|l|}{ Histological typing } \\
\hline Differentiated & $n=26$ & 17 & 9 & NS \\
\hline Undifferentiated & $n=25$ & 12 & 13 & \\
\hline \multicolumn{5}{|l|}{ Lymphatic invasion } \\
\hline ly $(-)$ & $n=10$ & 7 & 3 & NS \\
\hline ly $(+)$ & $n=39$ & $20(n=27)$ & 19 & \\
\hline \multicolumn{5}{|l|}{ Venous invasion } \\
\hline $\mathrm{v}(-)$ & $n=20$ & 15 & 5 & $P<0.05$ \\
\hline $\mathrm{v}(+)$ & $n=29$ & $12(n=27)$ & 17 & \\
\hline
\end{tabular}

Pathological diagnoses and classification of gastric cancer were made according to the Japanese classification of gastric carcinona ${ }^{12}$

University, between 1985 and 1994. We selected tissue samples from patients who had an invasion beyond the muscularis propria without distant metastases. No histological lymph node metastasis at the time of surgery was confirmed by conventional routine $\mathrm{H} \& \mathrm{E}$ staining. The clinicopathological profiles of the patients are shown in Table 1. Pathological diagnoses and classification of the gastric cancer were made according to the rules of the Japanese classification of gastric carcinoma [12]. All patients had undergone total or distal gastrectomy with D2 (dissection of all group 1 and group 2 nodes) or more extensive lymph node dissection. No patient had had chemotherapy before the operation, but most of the patients were treated with adjuvant chemotherapy after the operation. Clinical information was available for all patients, and the mean follow-up period was 53.8 months (range, 1-120 months).

All specimens were fixed in formalin and embedded in paraffin, sliced at 5- $\mu \mathrm{m}$ thickness, and attached to glass slides. One section from each lymph node was stained with H\&E. We diagnosed no lymph node metastasis in the H\&E sections. One additional serial section from each lymph node was stained immunohistochemically, using AE1/AE3 (20:1 mixture of AE1 to AE3; IgG1, Boehringer Mannheim, Mannheim, Germany), a pool of monoclonal antibodies reactive to a broad spectrum of human keratins [13]. Tissue sections were rehydrated. The endogenous peroxidase activity 
was inhibited with $3 \%$ hydrogen peroxide in methanol. Before being stained, the sections were pretreated for $30 \mathrm{~min}$ with $0.1 \%$ trypsin in Tris-buffered saline with $0.1 \%$ calcium chloride. Non-specific background staining was inhibited with normal horse serum for $20 \mathrm{~min}$. The sections were then incubated with $10 \mu \mathrm{g} / \mathrm{ml}$ of AE1/AE3 for $120 \mathrm{~min}$, with biotin-conjugated horse immunoglobulin to mouse immunoglobulins for $30 \mathrm{~min}$, and finally with avidin-biotinylated peroxidase complex for 30 min (Vectastain ABC-PO kit; Vector Laboratories, Burlingame, CA, USA). Peroxidase activity was shown with diaminobendizine. Nuclear staining was shown with Mayer's hematoxylin. For a positive control, tissue sections of positive lymph node were included. Negative control sections were stained without primary antibody.

We examined 1390 lymph nodes (mean, 27.3 nodes per patient) from the 51 gastrectomy patients. Microinvolvement was defined as the presence of cytokeratin-positive cells in the regional lymph nodes of the stomach. We classified the grade of microinvolvement into three groups: grade 0 (G0), no positive cells in the lymph node; grade 1 (G1), fewer than four discrete positive cells; and grade 2 (G2), more than five positive cells or cell clusters. H\&E staining alone did not identify these positive tumor cells.

The $\chi^{2}$ test was performed to evaluate the relationship between microinvolvement and clinicopathological factors. Survival data were calculated with the KaplanMeier method model and the log rank test was used to assess significant differences between the groups. The level of significance was $P<0.05$.

\section{Results}

A single cytokeratin-positive cell in a negative node defined by $\mathrm{H} \& \mathrm{E}$ staining (G1) is shown in Fig. 1 (low magnification) and Fig. 2 (high magnification). Cytokeratin-positive cells showed diffuse staining in the cytoplasm, with intense staining in the cytoplasmic membrane. Figure 3 shows G2 microinvolvement in which more than five cytokeratin-positive cells were observed. Figure 4 also shows G2 microinvolvement, in which cytokeratin-positive cells appeared as small clusters. Of the 1390 lymph nodes that were not confirmed as metastases by $\mathrm{H} \& \mathrm{E}$ staining examination, 67 (4.9\%) had cytokeratin-positive cells, which consisted of G1 microinvolvement in 38 lymph nodes $(2.7 \%)$ and G2 in $29(2.1 \%)$. The incidence of positive nodes per patient varied considerably, from 1 to 12 . Of the 67 lymph nodes with microinvolvement, 51 were group $1 ; 14$, group 2; and 2, group 3. Skip microinvolvement to distant nodes (group 2 or group 3) was found in a few negative perigastric nodes (group 1).

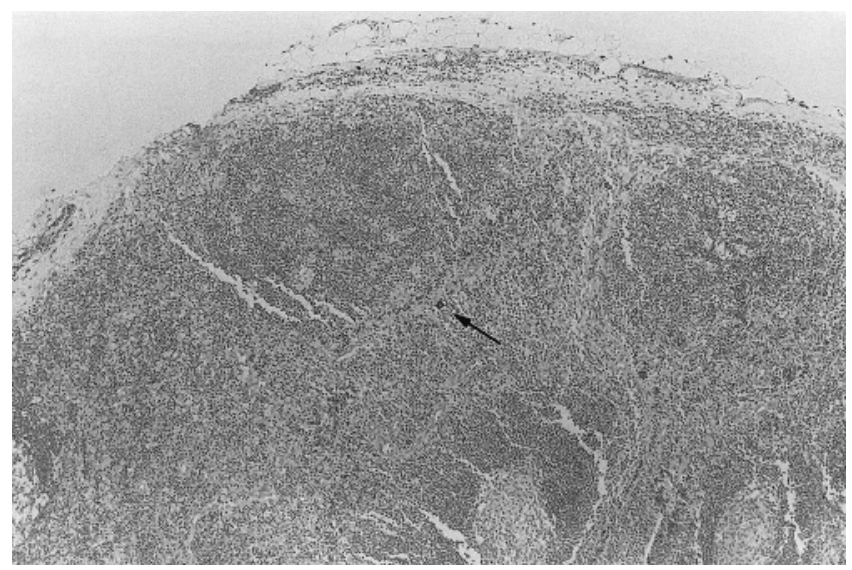

Fig. 1. Grade 1 microinvolvement (see text for explanation of grade of microinvolvement). A single atypical cell in the lymph node stained strongly for cytokeratin. $\times 40$

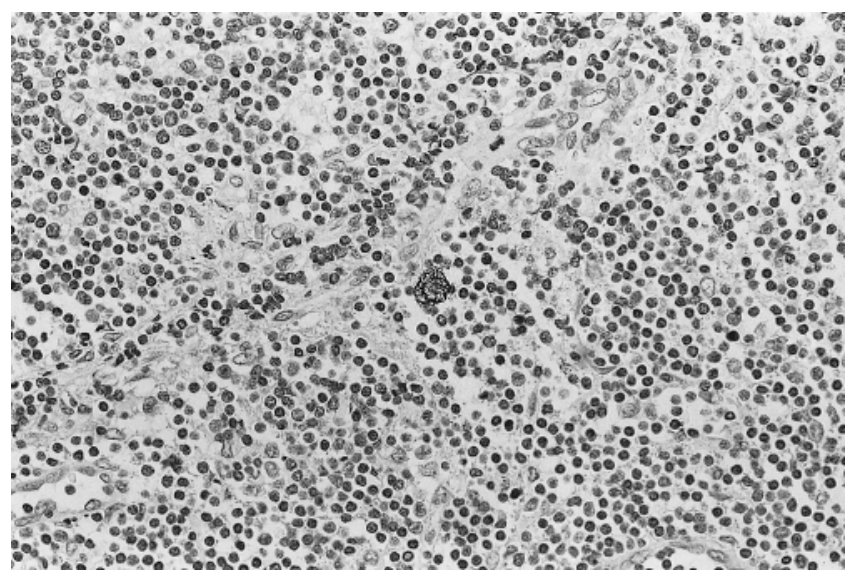

Fig. 2. Grade 1 microinvolvement. $\times 200$

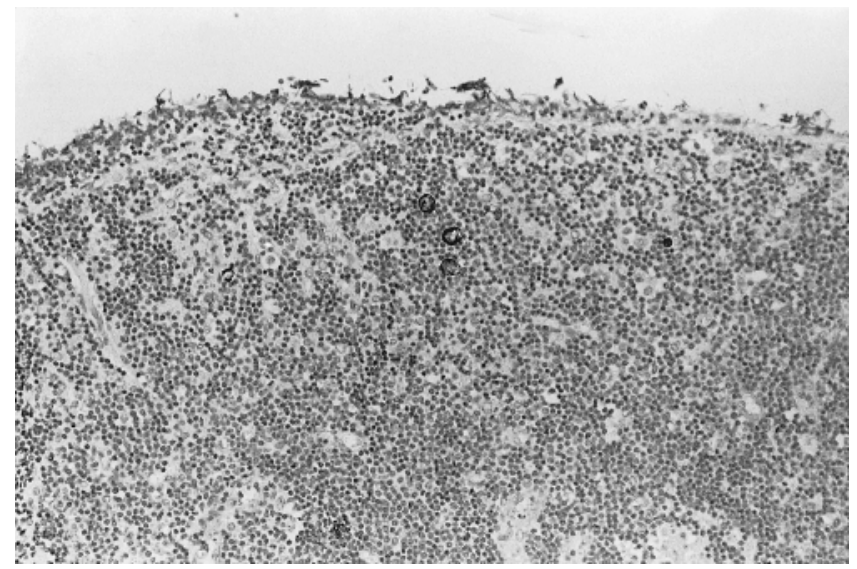

Fig. 3. Grade 2 microinvolvement. More than five cells stained strongly for cytokeratin. $\times 200$ 


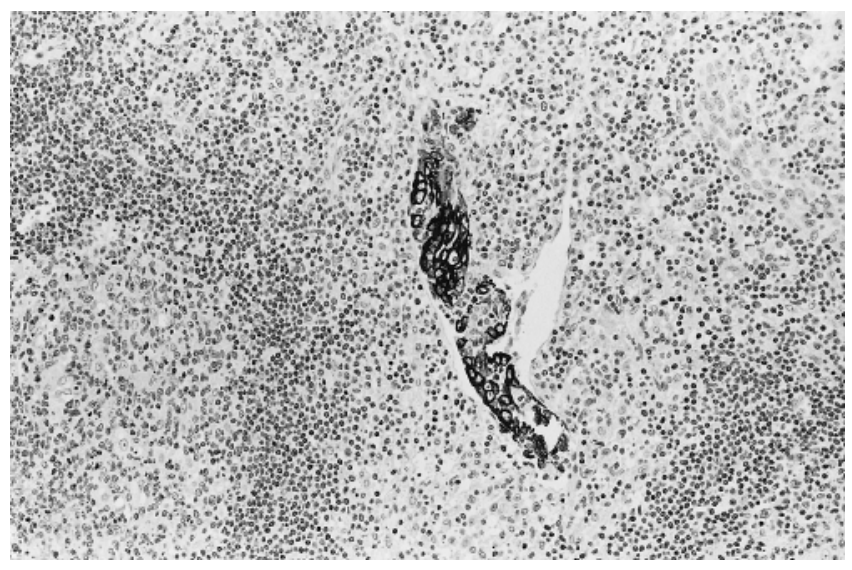

Fig. 4. Grade 2 microinvolvement. Positive cells that stained strongly for cytokeratin formed clusters. $\times 200$

Of the 51 patients with gastric cancer without histologically positive lymph nodes, immunohistochemical microinvolvement was identified in $22(43.2 \%)$. In regard to the relationship between microinvolvement and various tumor characteristics, microinvolvement increased in accordance with the maximum size of the tumor. In all 4 patients with tumors of more than $80 \mathrm{~mm}$, microinvolvement was identified. The incidence of microinvolvement also increased in accordance with the depth of tumor invasion. Thirteen of 39 patients (33.3\%) with depth of invasion within the subserosa ( $\mathrm{t} 2$ ) showed microinvolvement. In contrast, 9 of 12 patients $(75 \%)$ with invasion penetrating the serosa or further into the organ $(\mathrm{t} 3, \mathrm{t} 4)$ had microinvolvement. There was no correlation between microinvolvement and patient's sex or age, or the location or histological type of the tumor.

The clinical outcome of patients with and without microinvolvement was not significantly different (Fig. 5). Three patients died of peritoneal dissemination during the follow-up period. These 3 patients were immunohistochemically diagnosed as having been lymph-node positive. Two of the 3 patients with recurrence were diagnosed with grade 1 microinvolvement, and one of the 3 patients was diagnosed with grade 2 . The maximum tumor size in 1 patient was more than $90 \mathrm{~mm}$, and that in the other 2 patients was $40-80 \mathrm{~mm}$. Depth of invasion was $t 2$ in 2 patients and $t 3$ in 1 . There were no significant differences between patients with recurrence and those with no recurrence. Of the 29 patients who had no microinvolvement, 2 died of other causes. No patients died of cancer metastasis within the observation period. Figure 6 shows the Kaplan-Meier survival curves for patients with and without microinvolvement in regional lymph nodes who died of recurrence. The difference in survival between those with

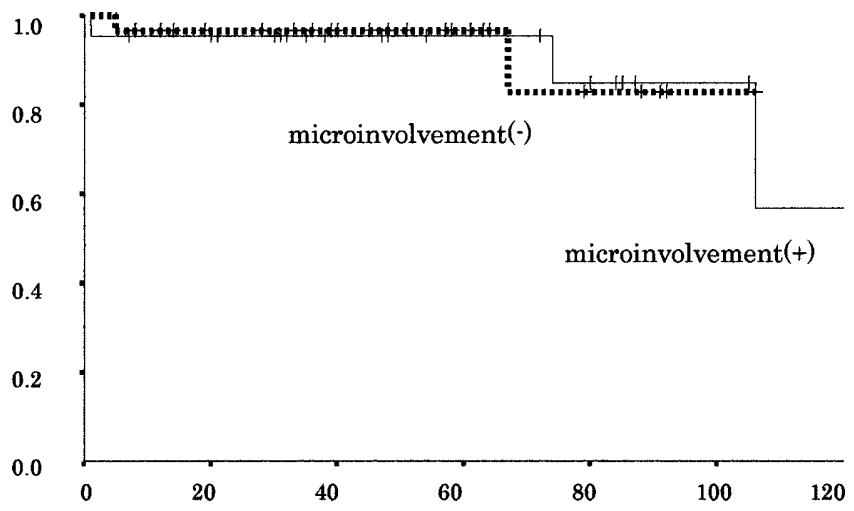

Fig. 5. Kaplan-Meier survival curves, including overall deaths, for patients with node-negative gastric cancer with and without microinvolvement in regional lymph nodes shown by cytokeratin antibody $(P=0.845)$

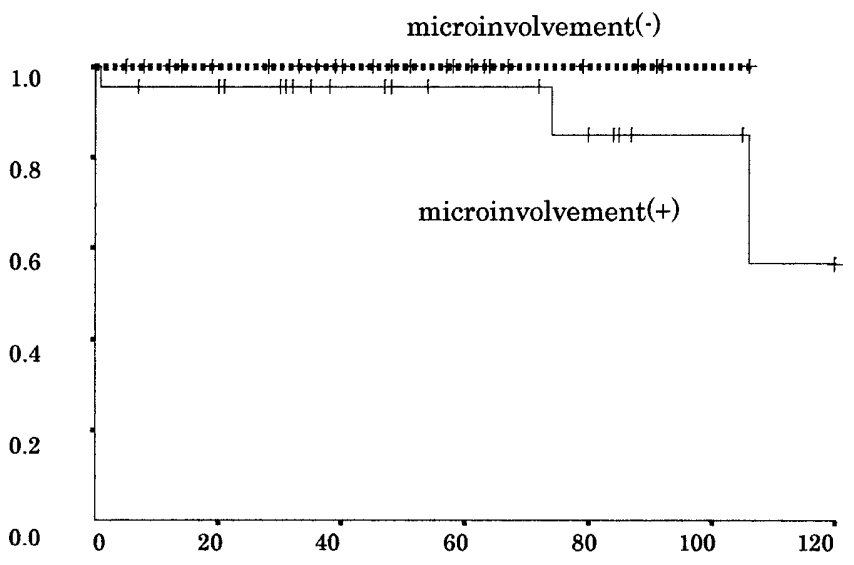

Fig. 6. Kaplan-Meier survival curves for patients with nodenegative gastric cancer with and without microinvolvement in regional lymph nodes, shown by cytokeratin antibody, who died of recurrence $(P=0.15)$

and without microinvolvement was not significant $(P=0.15)$.

\section{Discussion}

The monoclonal antibody mixture AE1-AE3 is a pool of monoclonal antibodies reactive to a broad spectrum of human keratins in epithelial cells [13]. We performed an immunohistochemical study of 1390 lymph nodes dissected from human gastric cancer that were without lymph node metastasis detectable by conventional histological examination. We used antibodies against cytokeratin to detect microinvolvement. Micrometastases within lymph nodes from breast carcinoma $[8,14,15]$ and colorectal carcinoma [6,7,16-18] have been detected immunohistochemically by using anti- 
bodies to early membrane antigen (EMA), cytokeratin, carcinoembryonic antigen (CEA), and/or S-100 protein. Cytokeratin is a specific marker among antibodies referred as specific epithelial cell markers $[9,10,11]$. Small numbers of cancer cells in lymph nodes have sometimes been missed by routine histological methods, but immunostaining with cytokeratin antibody has made it possible to detect even a single tumor cell among thousands of normal cells [19].

We found microinvolvement in $4.8 \%$ of the resected lymph nodes and in $43.2 \%$ of the patients with nodenegative gastric cancer whose tissues we examined. Siewert et al. [11] reported microinvolvement in 56 of 62 patients with pN0 gastric cancer $(90 \%)$, by an immunohistological method using cytokeratin antibody. Maehara et al. [10] reported microinvolvement in 15 of $420(3.6 \%)$ nodes and in 8 of 34 patients $(23.5 \%)$ with cytokeratin-positive cells in the lymph nodes of nodenegative early gastric cancer. Ishida et al. [9] has reported that 197 of 2446 lymph nodes $(8.1 \%)$ had micrometastases identified by immunostaining for cytokeratin; however, these included samples from node-positive patients. Thus, a substantial proportion of negative H\&E-stained nodes is considered to have micrometastases.

It has been reported that meticulous analysis with serial sectioning of whole lymph nodes in which only $\mathrm{H} \& \mathrm{E}$ staining was done resulted in the detection of micrometasis in about $20 \%$ of node-negative patients examined by a routine method [20]. The detection rate of metastases within lymph nodes increases if the number of sections increases. However, it is of no use in routine practice to cut multiple serial sections from all lymph nodes. Adell et al. [21] reported that $95 \%$ of patients with micrometastases were identified by studying three sections from each of three lymph nodes of colorectal cancer. Therefore, studying only one section may be insufficient for detecting micrometastases. Recently, reverse transcriptase-polymerase chain reaction (RT-PCR) methods have been reported as more sensitive and have been extensively utilized to detect micrometastases in gastric cancer [22], breast cancer [23], and colorectal cancer [24].

As lymph node metastasis is likely to occur together with tumor progression, microinvolvement in gastric cancer increases in accordance with the size of tumor, depth of invasion, and venous invasion. Ishida et al. [9] reported that micrometastases were observed more frequently in diffuse-type gastric cancer, but these results were not found in our study.

Maehara et al. [10] reported that the survival of a cytokeratin-positive group of patients with early gastric cancer was significantly shorter than that of a cytokeratin-negative group. The presence of micrometastases in lymph nodes has been shown to be an indis- pensable factor in determining the prognosis of gastric cancer patients [9]. In this study, we found no difference in the survival of gastric cancer patients with and without microinvolvement. This is partly because the number of tumors and the follow-up period were limited. However, it must be noted that no patients without microinvolvement died of recurrence. Thus, determination of microinvolvement within lymph nodes is considered to be a more reliable prognostic factor than diagnosing lymph node involvement by conventional methods.

Regional lymph nodes are routinely dissected during gastric cancer surgery. However, it is not clear why many lymph nodes have to be dissected. Baba et al. [25] reported a higher recurrence rate in patients treated with $\mathrm{D} 1$ lymph node dissection than in those who received D2/3 dissection, even in patients with nodenegative early gastric cancer. This report suggests the possible presence of micrometastases in lymph nodes of node-negative gastric cancer.

It is uncertain whether cytokeratin-positive cells in lymph nodes will manifest as clinical involvement. During the formation of metastases in lymph nodes, cancer cells are thought to migrate in the lymph vessels, reach the lymph nodes, lodge in the sinus, and proliferate. Chronologically, G1 microinvolvement may mean that the cancer cells have just reached the lymph node, while G2 microinvolvement indicates the initiation of proliferation. However, it is not known how long after their initial arrival in the lymph nodes the cancer cells begin to proliferate, and it is also not known whether tumor cells in lymph nodes are really able to migrate and successfully colonize other organs.

Patients with bone marrow cytokeratin-positive cells showed a significantly shorter disease-free survival than those without cytokeratin-positive cells, but not all patients who had bone marrow tumor cells died [5]. Pantel et al. [26] reported that cytokeratin-positive cells existed in bone marrow, but it is still unclear whether these cells can proliferate or whether they are removed by the host immune response. The possibility of tumor cells proliferating and showing metastasis depends on various factors, such as cell viability, cell characteristics, the host immune response, and the microenvironment. It is not clear whether the cytokeratin-positive cells could grow to macroscopic metastases, but microinvolvement of at least $\mathrm{G} 2$ is regarded as metastasis. Further investigations with a greater number of patients and a longer follow-up period will clarify these problems.

\section{References}

1. Moriguchi S, Hayashi Y, Nose Y, Maehara Y, Korenaga D, Sugimachi $\mathrm{K}$, et al. A comparison of the logistic regression and 
the Cox proportional hazard models in retrospective studies on the prognosis of patients with gastric cancer. J Surg Oncol 1993; 52:9-13.

2. Adachi Y, Mori M, Maehara Y, Kitano S, Sugimachi K. Prognostic factors of node-negative gastric carcinoma: univariate and multivariate analyses. J Am Coll Surg 1997;184:373-7.

3. Moll R, Franke WW, Schiller DL, Geiger B, Krepler R. The catalog of human cytokeratins: patterns of expression in normal epithelia, tumors and cultured cells. Cell 1982;31:11-24.

4. Debus E, Moll R, Franke WW, Weber K, Osborn M. Immunohistochemical distinction of human carcinomas by cytokeratin typing with monoclonal antibodies. Am J Pathol 1984;114:121-30.

5. Lindemann F, Shlimok G, Dirschedl P, Witte J, Riethmuller G. Prognostic significance of micrometastatic tumor cells in bone marrow of colorectal cancer patients. Lancet 1992;340:685-9.

6. Cutait R, Alves VAF, Lopes LCL, Cutait DE, Borges JLA, Singer J, et al. Restaging of colorectal cancer based on the identification of lymph node micrometastases through immunoperoxidase staining of CEA and cytokeratins. Dis Colon Rectum 1991;34:917-20.

7. Greenson JK, Isenhart CE, Rice R, Mojzisik C, Houchens D, Martin E, et al. Identification of occult micrometastases in pericolic lymph nodes of Dukes'B colorectal cancer patients using monoclonal antibodies against cytokeratin and CC49. Cancer 1994;73:563-9.

8. Trojani M, de Mascarel I, Bonichon F, Coindre JM, Delsol G. Micrometastases to axillary lymph nodes from carcinoma of breast: detection by immunohistochemistry and prognostic significance. Br J Cancer 1987;55:303-6.

9. Ishida K, Katsuyama T, Sugiyama A, Kawasaki S. Immunohistochemical evaluation of lymph node micrometastases from gastric carcinomas. Cancer 1997;79:1069-76.

10. Maehara Y, Oshiro T, Endo K, Baba H, Oda S, Ichiyoshi Y, et al. Clinical significance of occult micrometastasis lymph nodes from patients with early gastric cancer who died of recurrence. Surgery 1996;119:397-402.

11. Siewert JR, Kestlmeier R, Busch R, Bottcher K, Roder JD, Müller J, et al. Benefits of D2 lymph node dissection for patients with gastric cancer and $\mathrm{pN} 0$ and $\mathrm{pN} 1$ lymph node metastases. $\mathrm{Br}$ J Surg 1996;83:1144-7.

12. Japanese Gastric Cancer Association. Japanese classification of gastric carcinoma 2nd English ed. Gastric Cancer 1998;1:10-24.

13. Woodcock-Mitchell J, Eichner R, Nelson WG, Sun TT. Immunolocalization of keratin polypeptides in human epidermis using monoclonal antibodies. J Cell Biol 1982;95:580-8.

14. Sloane JP, Ormerd MG, Imrue SF, Coombes RC. The use of antisera to epithelial membrane antigen in detecting micro- metastases in histological sections. Br J Cancer 1980;42:3928.

15. Tsuchiya A, Sugano K, Kimijima I, Abe R. Immunohistochemical evaluation of lymph node micrometastases from breast cancer. Acta Oncol 1996;35:425-8.

16. Crowson M, Hockey MS, Newman J, Stokes H, Macdonald F, Fielding JW, et al. An immunocytochemical study of carcinoembryonic antigen (CEA) expression in colorectal tumors and their metastases using a monoclonal antibody. Br J Surg 1984;5:376.

17. Makin CA, Bobrow LG, Nicholls RJ. Can immunohistology improve detection of lymph-node metastases in large-bowel cancer? Dis Colon Rectum 1989;32:99-102.

18. Haboubi NY, Clark P, Kaftan SM, Schofield PF. The imprtance of combining xylene clearance and immunohistochemistry in the accurate staging of colorectal carcinoma. J R Soc Med 1992; 85:386-8.

19 Hayashi N, Ito I, Yanagisawa A, Kato Y, Nakamori S, Imaoka S, et al. Genetic diagnosis of lymph-node metastasis in colorectal cancer. Lancet 1995;345:1257-9.

20. Gusterson B. Are micrometastases clinically relevent? Br J Hosp 1991;47:247-8.

21. Adell G, Boeryd B, Franlund B, Sjodahl R, Hakansson L. Occurrence and prognostic importance of micrometastases in regional lymph nodes in Dukes B colorectal carcinoma: an immunohistochemical study. Eur J Surg 1996;162:637-42.

22. Noguchi S, Hiratsuka M, Furukawa H, Aihara T, Kasugai T, Tamura S, et al. Detection of gastric cancer micrometastases in lymph nodes by amplification of keratin 19 mRNA with reverse transcriptase-polymerase chain reaction. Jpn J Cancer Res 1996;87:650-4.

23. Noguchi S, Aihara T, Motomura K, Inaji H, Imaoka S, Koyama $\mathrm{H}$, et al. Detection of breast cancer micrometastases in axillary lymph nodes by means of reverse transcriptase-polymerase chain reaction. Comparison between MUC1 mRNA and keratin 19 mRNA amplification. Am J Pathol 1996;148:649-56.

24. Gunn J, McCall JL, Yun K, Wright PA. Detection of micrometastases in colorectal cancer patients by K19 and K20 reversetranscription polymerase chain reaction. Lab Invest 1996;75: 611-6.

25. Baba H, Maehara $\mathrm{Y}$, Takeuchi $\mathrm{H}$, Inutsuka S, Okuyama $\mathrm{T}$, Yosuke A, et al. Effect of lymph node dissection on the prognosis in patients with node-negative early gastric cancer. Surgery 1995;117:165-9.

26. Pantel K, Schlimok G, Braun S, Kutter D, Lindemann F, Schaller $\mathrm{G}$, et al. Differential expression of proliferation-associated molecules in individual micrometastatic carcinoma cells. J Natl Cancer Inst 1993;85:1419-23. 